\title{
Prüfer Conditions in Commutative Rings
}

\section{Sarah Glaz \& Ryan Schwarz}

\section{Arabian Journal for Science and Engineering}

ISSN 1319-8025

Volume 36

Number 6

Arab J Sci Eng (2011) 36:967-983

DOI 10.1007/s13369-011-0049-5
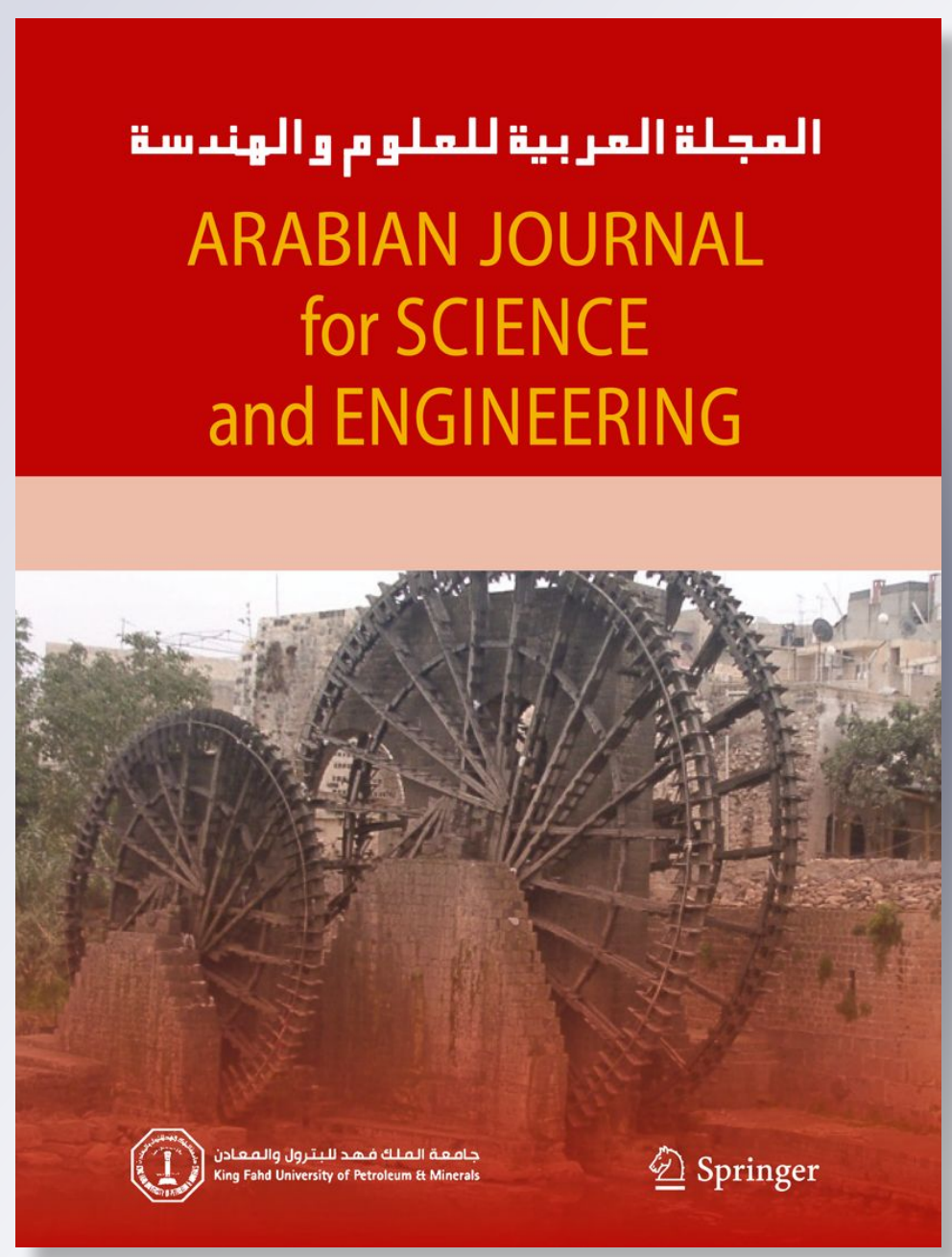

算 Springer 
Your article is protected by copyright and all rights are held exclusively by King Fahd University of Petroleum and Minerals. This e-offprint is for personal use only and shall not be self-archived in electronic repositories. If you wish to self-archive your work, please use the accepted author's version for posting to your own website or your institution's repository. You may further deposit the accepted author's version on a funder's repository at a funder's request, provided it is not made publicly available until 12 months after publication. 
RESEARCH ARTICLE - MATHEMATICS

Sarah Glaz • Ryan Schwarz

Prüfer Conditions in Commutative Rings

Received: 29 December 2010 / Accepted: 6 April 2011 / Published online: 6 July 2011

(C) King Fahd University of Petroleum and Minerals 2011

Abstract This article explores several extensions of the Prüfer domain notion to rings with zero divisors. These extensions include semihereditary rings, rings with weak global dimension less than or equal to 1 , arithmetical rings, Gaussian rings, locally Prüfer rings, strongly Prüfer rings, and Prüfer rings. The renewed interest in these properties, due to their connection to Kaplansky's Conjecture, has resulted in a large body of results shedding new light on the area. We survey the work done in this direction in the last 15 years, including results, examples and counterexamples, and a multitude of open problems.

Keywords Semihereditary ring - Ring with global weak dimension at most 1 . Arithmetical ring . Gaussian ring · Prüfer ring

Mathematics Subject Classification (2000) $13 \mathrm{~F} 05 \cdot 13 \mathrm{D} 02 \cdot 13 \mathrm{D} 05$

\section{Introduction}

In his article "Untersuchungen über Teilbarkeitseigenshaften in Korpern" [47], Heinz Prüfer introduced a new class of integral domains, namely those domains $R$ in which all finitely generated non-zero ideals are invertible. In 1936, 2 years after Prüfer's untimely death, Krull [37] named these rings in Prüfer's honor and proved the first of the many equivalent conditions that make integral domains Prüfer. Since then, as noted in Glimer's volume [24], Prüfer domains have played a central role in non-Noetherian commutative algebra. Scattered

S. Glaz $(\bowtie) \cdot$ R. Schwarz

Department of Mathematics, University of Connecticut, Storrs, CT 06269, USA

E-mail: sarah.glaz@uconn.edu

R. Schwarz

E-mail: ryan.schwarz@uconn.edu 
among a number of chapters in Gilmer's volume [24] one counts no less than 22 equivalent definitions for a Prüfer domain. Bazzoni and Glaz [6] display the entire collection as one theorem. One of the equivalent characterizations of a Prüfer domain appearing in this collection is a notion defined by Tsang [51] that is applicable in a general ring setting. This is the notion of a Gaussian ring (see definition in Sect. 2). The notion of a Gaussian ring provided one of the first extensions of the Prüfer domain notion to rings with zero divisors. This notion also provided the motivation for the renewed investigation into other Prüfer conditions in a general ring setting. This article surveys the work done in this direction in the last 15 years, including results, examples and counterexamples, and the multitude of open problems in the area that still remain unanswered.

The article is roughly divided into three parts. The first part, comprising of Sects. 2-4, provides background, motivation, and basic results and examples in the area. Thus Sect. 2 starts with Kaplansky's Conjecture and the efforts made by many researchers to solve it. This section leads to Sect. 3, which contains the definitions and basic properties of six Prüfer conditions in general ring setting. Section 4 provides the basic relations between the six properties and opens the way to the deeper investigations that followed. The second part consists of Sects. 5 and 6. Sect. 5 elaborates on the role which certain zero-divisor conditions and total ring of quotient behavior played in clarifying the relations between the six Prüfer conditions. Section 6 highlights the results and open questions related to the behavior of homological dimensions in rings possessing one of the Prüfer conditions. The third part of this article, Sects. 7 and 8, describes the work done in ring constructions. Sect. 7 elaborates on the results obtained in pullback rings, while Sect. 8 introduces two special localizations of polynomial rings, as well as a seventh Prüfer condition, and the beginning of a possible investigation of their behavior under any of the seven Prüfer conditions.

Throughout this paper all rings are commutative with unit, all modules are unital, and a local ring means a, not necessarily Noetherian, ring with a unique maximal ideal.

\section{Motivation: Kaplansky's Conjecture and the Gaussian Condition}

Let $R$ be a commutative ring and let $f \in R[x]$ be a polynomial in one variable over $R$. The content of $f$, denoted by $c(f)$, is the ideal of $R$ generated by the coefficients of $f$. The content of a polynomial, $c(f)$, satisfies a number of multiplicative properties. For example, it follows immediately that for every two polynomials $f$ and $g$ in $R[x]$ :

$$
c(f g) \subset c(f) c(g) .
$$

In fact, the Dedekind-Mertens Lemma (see, for example, $[3,23]$ ) asserts that for every two polynomials $f$ and $g$ in $R[x]$ :

$$
c(f)^{n} c(f g)=c(f)^{n+1} c(g), \text { where } n=\operatorname{deg} g .
$$

Definition 2.1 (Tsang 1965 [51]) Let $f \in R[x]$. If $c(f g)=c(f) c(g)$ for every $g \in R[x]$, then $f$ is called a Gaussian polynomial. Moreover, if $c(f g)=c(f) c(g)$ for every pair of polynomials $f$ and $g \in R[x]$, then $R$ is called a Gaussian ring.

In her 1965 thesis, Tsang determined conditions under which a polynomial is Gaussian. Specifically:

Theorem 2.2 ([51]) Let $R$ be a commutative ring and let $f \in R[x]$ be a polynomial in one variable over $R$. If $c(f)$ is an invertible ideal, or more generally, a locally principal ideal, then $f$ is a Gaussian polynomial.

The converse of this statement has received a considerable amount of attention in the recent past and is the content of the following conjecture of Kaplansky.

Kaplansky's Conjecture Let $R$ be a commutative ring and let $f \in R[x]$ be a Gaussian polynomial. Then $c(f)$ is an invertible or, at least, a locally principal ideal.

A number of authors contributed to the solution of this conjecture: Anderson D.D. and Kang (1995 [2]) initiated the renewed investigation into this conjecture; Glaz and Vasconcelos (1997 [27], 1998 [28]) showed that the conjecture holds for integrally closed Noetherian domains; Heinzer and Huneke (1997 [34]) proved that the conjecture holds for Noetherian domains; Loper and Roitman (2005 [39]) solved the conjecture affirmatively for all domains; Lucas (2005 [42]) extended this solution to a partial answer for non-domains. The following theorem summarizes the combined results obtained by these authors. 
Theorem 2.3 ([2,27,28,34,39,42,51]) Let $R$ be a commutative ring and let $f \in R[x]$ be a Gaussian polynomial. The following hold:

(1) If $(0: c(f))=0$, then $c(f)$ is locally principal.

(2) If $c(f)$ is a regular ideal, then $c(f)$ is invertible.

(3) In particular, if $R$ is a domain, then $c(f)$ is invertible.

In general, however, Kaplansky's Conjecture is false (see [27,28]).

Example $2.4[27,28]$ A Gaussian polynomial $f$ with $c(f)$ not locally principal.

Let $k$ be a field and let $t$ and $u$ be indeterminates over $k$. Set $R=k[t, u] /(t, u)^{2}=k[T, U]$, where $T=t+(t, u)^{2}$ and $U=u+(t, u)^{2}$, and let $f=T x^{2}+U x+T \in R[x]$. Then $f$ is Gaussian and $c(f)=(T, U)$ is not principal. In fact, $R$ is a Gaussian local ring with maximal ideal $m=(T, U)$ and $m^{2}=0$.

Recently, progress has been made in solving this conjecture under some restrictions on the ring $R$. Lucas (2008 [43]) provided an answer when the ring is reduced:

Theorem 2.5 ([43]) Let $R$ be a reduced ring and let $f \in R[x]$ be a Gaussian polynomial. Then $c(f)$ is locally principal.

Bakkari, Kabbaj and Mahdou (2010 [5]) investigated Kaplansky's Conjecture for trivial ring extensions. Let $A$ be a ring and let $E$ be an $A$ module. The trivial ring extension of $A$ by $E$ is the ring $R=A \propto E$ whose underlying group is $A \times E$ with multiplication given by $(a, e)\left(a^{\prime}, e^{\prime}\right)=\left(a a^{\prime}, a e^{\prime}+a^{\prime} e\right)$. Since $(0, e)^{2}=0$ for any element $e$ in $E$, a trivial ring extension is never a reduced ring. Nevertheless, in certain cases the conclusion of Theorem 2.5 remains valid:

Theorem 2.6 ([5]) Let $R=A \propto K$ be the trivial ring extension of a domain A by its field of quotients $K$ and let $f \in R[x]$ be a Gaussian polynomial. Then $c(f)$ is locally principal.

Bakkari, Kabbaj and Mahdou [5] called a ring over which every Gaussian polynomial has locally principal content ideal, a pseudo-arithmetical ring. It will be interesting to characterize pseudo-arithmetical rings, and also to clarify the situation for general rings, namely:

Open Question 1 If $f$ is a Gaussian polynomial over a general ring $R$, with $(0: c(f)) \neq 0$, what can be said about $c(f)$ ? In particular, can one find necessary and sufficient conditions so that $c(f)$ is locally principal or invertible?

The answers that were obtained on the nature of the content ideal of a single Gaussian polynomial shed some, but not sufficient, light on the nature of Gaussian rings. At the same time, because these answers are incomplete, it was natural to try to understand the Gaussian notion better by investigating more thoroughly rings for which this condition holds for any pair of polynomials, that is, Gaussian rings. This was the motivating force behind the investigation initiated by Glaz in [29].

\section{Definitions: Six Prüfer Conditions for General Rings}

Let $R$ be a commutative ring and let $I$ be an ideal of $R$. Denote by $Q(R)=\left\{\frac{a}{b}: a, b \in R\right.$ and $b$ is a nonzero divisor of $R\}$ the total ring of quotients of $R$. Let $I^{-1}=\{r \in Q(R): r I \subset R\}$ be the inverse of $I$. Then $I$ is invertible if $I \cdot I^{-1}=R$. This definition agrees with the classical definition of invertibility of an ideal $I$ in an integral domain $R$ with field of quotients $Q(R)$.

We start with Prüfer's original definition for an integral domain $R$.

Definition 3.1 (Prüfer 1932 [47]) An integral domain $R$ is called a Prüfer domain if every finitely generated non-zero ideal of $R$ is invertible.

Prüfer also showed that to verify this condition, it suffices to check that it holds for all two-generated ideals of $R$.

Theorem 3.2 ([47]) An integral domain $R$ is a Prüfer domain if and only if every two-generated ideal of $R$ is invertible. 
Recall that $R$ is a valuation domain if for every element $x \in Q(R)$, the field of quotients of $R$, either $x \in R$ or $x^{-1} \in R$. Equivalently, the set of all the ideals of $R$ is totally ordered by inclusion. This is equivalent to saying that any two ideals are comparable. For a domain, this also means that the prime ideals of $R$ form a chain $p_{1} \subset p_{2} \subset \cdots$. In 1936, Krull proved the following theorem.

Theorem 3.3 ([37]) An integral domain $R$ is a Prüfer domain if and only if $R_{p}$ (respectively, $R_{m}$ ) is a valuation domain for every prime ideal $p$ of $R$ (respectively, maximal ideal $m$ of $R$ ).

The first Prüfer condition to be defined in a general ring setting is Fuchs' definition of an arithmetical ring.

Definition 3.4 (Fuchs 1949 [20]) A ring $R$ is an arithmetical ring if the ideals of $R_{m}$ are totally ordered by inclusion for all maximal ideals $m$ of $R$.

If $R$ is an integral domain, according to Krull's characterization, this condition is equivalent to $R$ being a Prüfer domain. There is another useful characterization of arithmetical rings due to Jensen.

Theorem 3.5 ([36]) A ring $R$ is arithmetical if and only if every finitely generated ideal of $R$ is locally principal.

Chronologically, the next Prüfer condition to be studied was Tsang's 1965 definition of a Gaussian ring (see Definition 2.1). Just as arithmetical domains are Prüfer domains, Gaussian domains are also Prüfer domains. This was proved independently by both Tsang and Gilmer, and is the statement of the following theorem.

Theorem 3.6 ([23,51]) Let $R$ be an integral domain. Then $R$ is a Prüfer domain if and only if $R$ is a Gaussian domain.

The Gaussian condition, similarly to the arithmetical condition, can be checked locally. In fact, there are a number of interesting characterizations of Gaussian local rings, some of which may be useful in further investigations. The following is a small collection of characterizations of Gaussian local rings.

Theorem 3.7 ([7,30,42,51]) Let $(R, m)$ be a local ring. The following conditions are equivalent:

(1) $R$ is a Gaussian ring.

(2) If $I$ is a finitely generated ideal of $R$, then $I / I \cap(0: I)$ is a cyclic $R$-module.

(3) If $I$ is a two-generated ideal of $R$, then $I / I \cap(0: I)$ is a cyclic $R$-module.

(4) For every two elements $a, b \in R$, there exists an element $d \in(0:(a, b))$ such that $(a, b)=(a, d)$ or $(a, b)=(b, d)$. Moreover, $d$ can be chosen so that $b \in d+a R$ or $a \in d+b R$.

(5) For every two elements $a, b \in R$, the following two properties hold:

(a) $(a, b)^{2}=\left(a^{2}\right)$ or $\left(b^{2}\right)$.

(b) If $(a, b)^{2}=\left(a^{2}\right)$ and $a b=0$, then $b^{2}=0$.

Tsang [51] proved the equivalence of (1), (2), and (3) and a connection between (1) and parts of (5). Glaz [30] showed that (1) implies (5) while Lucas [42] showed the reverse implication (5) implies (1). Bazzoni and Glaz [7] proved the equivalence of (1) and (4). In addition, Tsang showed that the prime ideals in a local Gaussian ring are totally ordered. As a consequence, $(R, m)$ has a unique minimal prime ideal $p=N$, the nilradical of $R$. It follows that $R / N$ is a reduced Gaussian ring, hence a valuation domain. Another consequence of this theorem is that if $R$ is Noetherian, then $(R, m)$ is Gaussian if and only if $m /(0: m)$ is cyclic, and this is equivalent to $R /(0: m)$ being arithmetical. This criterion is useful in constructing examples.

Another class of rings with connections to Prüfer conditions was introduced by Cartan and Eilenberg in 1956 [14] as a generalization of hereditary rings.

Definition 3.8 (Cartan and Eilenberg 1956 [14]) A ring $R$ is called a semihereditary ring if every finitely generated ideal of $R$ is projective.

In establishing the connection between semihereditary rings and Prüfer domains, we note that for an ideal $I$ of a ring $R$, there is a strong connection between invertibility, projectivity, and being locally principal.

Theorem 3.9 Let $R$ be a ring and let $I$ be an ideal of $R$.

(1) If I is invertible, then I is projective. 
(2) If I is projective, then I is locally principal.

(3) If I is finitely generated and regular then: I is invertible $\Leftrightarrow I$ is projective $\Leftrightarrow I$ is locally principal. In particular, all three conditions coincide when $R$ is a domain and $I$ is a finitely generated ideal.

As a direct consequence of Theorem 3.9 we obtain:

Corollary 3.10 $R$ is a Prüfer domain if and only if $R$ is a semihereditary domain.

Next, we will consider rings $R$ with weak global dimension less than or equal to one, denoted by w. $\operatorname{dim} R \leq 1$. The weak global dimension is the measure of flatness of modules over $R$. Rings with weak global dimension zero are those for which all modules over $R$ are flat. These are exactly the von Neumann regular rings (all of which are coherent). A few characterizations of von Neumann regular rings are given below, and can be found in $[25,50]$.

Theorem 3.11 Let $R$ be a ring. The following conditions are equivalent:

(1) $R$ is von Neumann regular.

(2) For every $x \in R$, there exists $y \in R$ such that $x^{2} y=x$.

(3) $R_{p}$ is a field for every prime ideal $p$ of $R$.

(4) Every finitely generated ideal of $R$ is principal, generated by an idempotent.

Von Neumann regular rings are special in that they are the closest among non-domains to behave like fields. Note that if w. $\operatorname{dim} R \leq 1$, then all ideals of $R$ are flat. This need not be the case for all $R$-modules, unless w. $\operatorname{dim} R=0$. The following result may be found in [25].

Theorem 3.12 Let $R$ be a ring. Then $\mathrm{w} . \operatorname{dim} R \leq 1$ if and only if $R_{p}$ is a valuation domain for every prime ideal $p$ of $R$.

As a consequence, domains $R$ with w. $\operatorname{dim} R \leq 1$ are Prüfer domains. In general, rings of w. $\operatorname{dim} R \leq 1$ are of two kinds: coherent and non-coherent.

Theorem 3.13 ([25,44]) Let $R$ be a ring. The following conditions are equivalent:

(1) $R$ is a semihereditary ring.

(2) w. $\operatorname{dim} R \leq 1$ and $R$ is a coherent ring.

(3) w. $\operatorname{dim} R \leq 1$ and w. $\operatorname{dim} Q(R)=0$.

(4) Every torsion-free $R$-module is flat.

(5) $Q(R)$ is von Neumann regular and $R_{p}$ is a valuation domain for every prime ideal $p$ of $R$.

The last Prüfer condition considered in [29] generalizes the notion of a Prüfer domain in the broadest sense. These are the so-called Prüfer rings introduced by Griffin in 1970 [33].

Definition 3.14 (Griffin 1970 [33]) A ring $R$ is a Prüfer ring if and only if every finitely generated regular ideal of $R$ is invertible.

Griffin proved many characterizations of Prüfer rings, but none that are particularly easy to utilize, this one included. There is an upside however. For domains, this definition clearly coincides with the notion of a Prüfer domain.

Definition 3.15 (Lucas 1986 [40]) A ring $R$ is called a locally Prüfer ring if $R_{p}$ is a Prüfer ring for every prime ideal $p$ of $R$.

If $R$ is a local Prüfer domain, then $R$ is a valuation domain. Therefore, by Krull's characterization, a domain $R$ is Prüfer if and only if it is locally Prüfer. The locally Prüfer condition first appeared in Lucas's 1986 article [40], which also provides an example of a Prüfer ring that is not locally Prüfer. The example below is due to Boynton [9]:

Example 3.16 [9] A Prüfer ring which is not locally Prüfer.

Let $A=k[w, x, y, z] /\left(w x, x^{2}, x y, x z\right)$, where $k$ is a field. Notice that $R=Q(A) \cong A_{m}$ where $m=$ ( $w, x, y, z)$. Since $R$ is a total ring of quotients, it is Prüfer (no ideal is regular), but $R$ has a localization that is a two-dimensional domain, so it is not locally Prüfer. In particular, for $p=(w, x, y), R_{p} \cong k(z)[x, y]_{(w, x, y)}$ is a two-dimensional Noetherian domain.

The results introduced in this section can be organized in the following table. The conditions in the first five rows of the left column are all equivalent. The relations between the conditions in the right column will be addressed in later sections. Placing the condition displayed in the bottom row on the total ring of quotients of a ring played an important role in the comparison between the other conditions in the right column (see Sect. 5). 


\begin{tabular}{|c|c|}
\hline Integral domain & General ring \\
\hline $\begin{array}{l}R \text { is Prüfer } \Leftrightarrow \text { every finitely generated ideal } \\
\text { of } R \text { is invertible }\end{array}$ & $\begin{array}{l}\text { Griffin (1970): } R \text { is a Prüfer ring } \Leftrightarrow \text { every } \\
\text { finitely generated regular ideal of } R \text { is } \\
\text { invertible }\end{array}$ \\
\hline $\begin{array}{l}R \text { is Prüfer } \Leftrightarrow R_{p} \text { is Prüfer for every prime } \\
\quad \text { ideal } p \text { of } R\end{array}$ & $\begin{array}{l}\text { Lucas (1986): } R \text { is locally Prüfer } \Leftrightarrow R_{p} \text { is } \\
\quad \text { Prüfer for every prime ideal } p \text { of } R\end{array}$ \\
\hline $\begin{array}{l}R \text { is Prüfer } \Leftrightarrow R \text { is Gaussian, i.e. } \\
\quad c(f g)=c(f) c(g) \text { for all } f, g \in R[x]\end{array}$ & Tsang (1965): $R$ is Gaussian \\
\hline $\begin{array}{l}\left.R \text { is Prüfer } \Leftrightarrow R_{p} \text { (resp., } R_{m}\right) \text { is a valuation } \\
\text { domain for every prime ideal } p \text { (resp., } \\
\text { maximal ideals } m \text { ) of } R \Leftrightarrow \text { ideals of } R_{p} \\
\left.\text { (resp., } R_{m}\right) \text { are totally ordered by } \\
\text { inclusion }\end{array}$ & $\begin{array}{l}\text { - Fuchs (1949): } R \text { is arithmetical } \Leftrightarrow \text { the } \\
\text { ideals of } R_{m} \text { are totally ordered by } \\
\text { inclusion for all maximal ideals } m \\
\text { - w. } \operatorname{dim} R \leq 1 \Leftrightarrow R_{p} \text { is a valuation } \\
\text { domain for every prime ideal } p \text { of } R\end{array}$ \\
\hline $\begin{array}{l}R \text { is Prüfer } \Leftrightarrow R \text { is semihereditary, i.e. the } \\
\text { finitely generated ideals of } R \text { are } \\
\text { projective }\end{array}$ & $\begin{array}{l}\text { Cartan and Eilenberg (1956): } R \text { is } \\
\text { semihereditary }\end{array}$ \\
\hline$R$ is a field & $\begin{array}{l}R \text { is von Neumann regular } \\
\quad \Leftrightarrow \text { w. } \operatorname{dim} R=0 \Leftrightarrow \text { for every } a \in R, \\
\text { there exists } b \in R \text { such that } a^{2} b=a\end{array}$ \\
\hline
\end{tabular}

\section{Basic Relations Between Prüfer Conditions}

In this section we will follow the first approach taken in [29] for understanding the relations between the six Prüfer conditions defined in the previous section in the general ring setting. The relations between these conditions can be expressed as follows:

(1) R semihereditary

$$
\subsetneq
$$

(2) $\quad$ w. $\operatorname{dim} R \leq 1$<smiles>[CH-]</smiles>

(3) $\mathrm{R}$ arithmetical

$$
\subsetneq
$$

(4) R Gaussian

$$
\subsetneq
$$

(5) R locally Prüfer

$$
\subsetneq
$$

(6) R Prüfer

It is possible to obtain reverse inclusions by imposing additional hypotheses, but this will be addressed in later sections. For now, we justify the above chain of proper inclusions, giving proofs and counterexamples.

\section{$R$ semihereditary $\subsetneq \mathrm{w} \cdot \operatorname{dim} R \leq 1$}

Recall from Theorem 3.13 that $R$ is a semihereditary ring if and only if $\mathrm{w} . \operatorname{dim} R \leq 1$ and $R$ is coherent. This gives the forward inclusion and also leads to a counterexample for the reverse containment. 
Example 4.1 A non-semihereditary ring with $\mathrm{w} \cdot \operatorname{dim} R \leq 1$.

Let $I$ be the ideal of $T=\prod \mathbb{Q}[x]$ generated by $f=\left(x, 0, x^{2}, 0, x^{3}, 0, \ldots\right)$ and let $D$ be the subring of $T$ consisting of all sequences that eventually consist of constants. Set $R=I+D$. $R$ is not coherent because $(0: f)$ is not a finitely generated ideal, and one can show that w. $\operatorname{dim} R$ is less than or equal to 1 . Note that since $R$ is not semihereditary, neither is $Q(R)$, because if $Q(R)$ were semihereditary, its total ring of quotients, which is equal to itself, must be von Neumann regular. This would imply that $R$ itself is semihereditary. Since w. $\operatorname{dim} Q(R) \leq$ w. $\operatorname{dim} R \leq 1$, this contradiction shows that any non-semihereditary ring of weak global dimension one has a total ring of quotients satisfying the same property.

\section{w. $\operatorname{dim} R \leq 1 \subsetneq R$ arithmetical}

Since w. $\operatorname{dim} R \leq 1$, by Theorem $3.12, R_{p}$ is a valuation domain for every prime ideal $p$. Therefore, every finitely generated ideal of $R$ is locally principal, hence $R$ is arithmetical. Jensen [36] found necessary and sufficient conditions for rings of weak global dimension at most one to be arithmetical rings.

Theorem 4.2 ([36]) A ring $R$ satisfies $\mathrm{w} . \operatorname{dim} R \leq 1$ if and only if $R$ is an arithmetical reduced ring.

This result allows the construction of an example of an arithmetical ring with w. $\operatorname{dim} R>1$.

Example 4.3 An arithmetical ring with w. $\operatorname{dim} R>1$.

Let $R=\mathbb{Z}_{4}$, the ring of integers modulo 4 . The only non-zero ideal of this ring is $2 \mathbb{Z}_{4}$, therefore $R$ is arithmetical. Note that $\mathbb{Z}_{4}$ is not reduced because $2^{2}=4=0$. By Theorem 4.2 w. dim $\mathbb{Z}_{4}>1$. Another example of such a ring is $R=k[u] /\left(u^{2}\right)$, where $k$ is a field and $u$ is an indeterminate. In this case $R=Q(R)$ and w. $\operatorname{dim} R=\infty$.

\section{$\boldsymbol{R}$ arithmetical $\subsetneq \boldsymbol{R}$ Gaussian}

Let $c(f)$ be the content ideal of a polynomial $f$ in $R[x]$, where $R$ is an arithmetical ring. Since $c(f)$ is finitely generated and $R$ is arithmetical, $c(f)$ is locally principal. By Theorem 2.2, we conclude that $f$ is Gaussian. It follows that $R$ is a Gaussian ring. This also leads to an example of a non-arithmetical Gaussian ring.

Example 4.4 A Gaussian ring that is not arithmetical.

The ring $R=k[t, u] /(t, u)^{2}$ of Example 2.4 is a Gaussian ring with a finitely generated ideal which is not locally principal. Specifically, the content ideal of $f=T x^{2}+U x+T$ is $c(f)=(T, U)$. Note that $R=Q(R)$.

\section{$R$ Gaussian $\subsetneq R$ locally Prüfer}

Let $R$ be a Gaussian ring, and let $I$ be a finitely generated regular ideal of $R$. Then $I=c(f)$ for some polynomial $f$ in $R[x]$, and by Theorem 2.3, $I$ is invertible. It follows that any Gaussian ring is a Prüfer ring. Since a Gaussian ring $R$ is locally Gaussian, it follows that every localization of a Gaussian ring $R$ is Prüfer. Therefore, $R$ is locally Prüfer.

Example 4.5 A locally Prüfer ring which is not Gaussian.

Let $R=k[t, u] /(t, u)^{3}$ where $k$ is a field. We have that $R=k[T, U]$ is a local ring with maximal ideal $m=(T, U)$ and $R=Q(R)$. So, $R$ is a Prüfer local ring. Since $m^{3}=0$ and $m$ is the unique prime ideal of $R$, it follows that $R$ is in fact locally Prüfer. It is not, however, Gaussian. One way to see this is to set $f=T x+U$ and $g=T x-U$ so that $f g=T^{2} x^{2}-U^{2}$. Then $c(f g)=\left(T^{2}, U^{2}\right) \neq\left(T^{2}, T U, U^{2}\right)=c(f) c(g)$.

\section{$\boldsymbol{R}$ locally Prüfer $\subsetneq \boldsymbol{R}$ Prüfer}

Let $I$ be a finitely generated regular ideal of $R$. Since $R$ is locally Prüfer, $I$ is locally invertible. An appeal to Theorem 3.9 gives us that $I$ itself is invertible, hence $R$ is a Prüfer ring. This inclusion was also proved by different methods in [9] and in [40]. An example of a Prüfer ring that is not locally Prüfer was given in Example 3.16.

\section{Conditions that Allow Reversal of Implications}

Given the characterizations of the six Prüfer conditions for general rings, it is natural to ask to what extent it is possible to reverse some or all of the implications. For instance, under what conditions is a Gaussian ring 
arithmetical, of weak global dimension at most one, or semihereditary? There are two distinct approaches to this question. The first approach, taken by Glaz in [30] utilizes conditions which control zero divisors. The second approach, taken by Bazzoni and Glaz in [7] and by Boynton in [10], consists of imposing conditions on $Q(R)$. We describe the former approach first, starting with a brief background on zero divisor controlling conditions.

Definition 5.1 A ring $R$ is a PF ring if every principal ideal of $R$ is flat. A ring $R$ is a PP ring if every principal ideal of $R$ is projective.

Both the PP and PF properties control zero divisors locally. The specific results are given below.

Theorem 5.2 ([45]) Let $R$ be a ring. The following conditions are equivalent:

(1) $R$ is a PF ring.

(2) $R_{p}$ is a domain for all prime ideals $p$ of $R$.

(3) $R_{m}$ is a domain for all maximal ideals $m$ of $R$.

(4) $R$ is reduced and every maximal ideal $m$ of $R$ contains a unique minimal prime $p$. In this case, $p=\{r \in R: \exists u \in R \backslash m$ such that $u r=0\}$ and $R_{p}=Q\left(R_{m}\right)$.

Even though both the PP and the PF conditions are homological in nature, the PP condition has a couple of ring theoretic characterizations.

Theorem 5.3 Let $R$ be a ring. The following conditions are equivalent:

(1) $R$ is a PP ring.

(2) $(0: a)$ is generated by an idempotent for every $a \in R$.

(3) Every element $r \in R$ can be expressed as $r=u e$ where $u$ is a nonzero divisor and e is an idempotent.

One can also control zero divisors by requiring $Q(R)$ to be von Neumann regular. The three conditions are connected by the following result, proved in 1971 by Quentel [48], and adjusted for correctness in 1989 by Glaz in [25].

Theorem 5.4 ([25,48]) Let $R$ be a ring. The following conditions are equivalent:

(1) $R$ is a PP ring.

(2) $R$ is a $P F$ ring and $Q(R)$ is a von Neumann regular ring.

(3) $R$ is a PF ring and Min $(R)$ is compact.

Here $\operatorname{Min}(R)$ denotes the set of all minimal prime ideals of $R$. $\operatorname{Min}(R)$ is a subspace of Spec $R$ in the Zariski topology. A more extensive exposition on the relations between the conditions mentioned in Theorem 5.4 can be found in [25]. This includes examples illustrating the independence between the four conditions mentioned in the previous theorem.

These zero divisor controlling conditions allow us to describe when Gaussian rings are semihereditary rings or of weak global dimension at most one.

Theorem 5.5 ([30]) Let $R$ be a ring. The following conditions are equivalent:

(1) $R$ is semihereditary.

(2) $R$ is a Gaussian ring and a $P P$ ring.

(3) $R$ is a Gaussian ring and $Q(R)$ is a von Neumann regular ring.

Theorem 5.6 ([30]) Let $R$ be a ring. The following conditions are equivalent:

(1) $\quad$ w. $\operatorname{dim} R \leq 1$.

(2) $R$ is a Gaussian ring and a PF ring.

(3) $R$ is a Gaussian ring and a reduced ring.

As a bonus, these results establish a couple of equivalences between zero divisor controlling conditions that do not hold in general. In particular, for a Gaussian ring $R$, the PP and $Q(R)$ von Neumann regular conditions are equivalent. Additionally, the PF condition is equivalent to the ring being reduced in the Gaussian context.

The other approach to reversing the implications of Prüfer conditions involves restrictions on the total ring of quotients. The idea is to start with a "weaker" property on $R$ and impose a "stronger" property on $Q(R)$. For instance, condition 3 of Theorem 5.5 which requires $R$ to be Gaussian and $Q(R)$ to be von Neumann regular; together these two conditions imply that $R$ is a semihereditary ring. It should also be noted that if $R$ is a domain, that is, $Q(R)$ is a field, then all of the Prüfer conditions are equivalent. The first step of this approach was to determine if $Q(R)$ inherits the Prüfer condition of $R$. Conditions 1-4 and 6 are treated in [7], while condition 5 is treated in [10]. Below is the complete picture: 
(a) If $R$ is a semihereditary ring, then $Q(R)$ is von Neumann regular, and hence semihereditary.

(b) If w. $\operatorname{dim} R \leq 1$, then w. $\operatorname{dim} Q(R) \leq 1$, since $Q(R)$ is a localization of $R$.

(c) If $R$ is an arithmetical ring, then $Q(R)$ is arithmetical by the following theorem of Jensen [36]:

Theorem 5.7 ([36]) Let $R$ be an arithmetical ring. Then any ring between $R$ and $Q(R)$ is an arithmetical ring.

(d) If $R$ is a Gaussian ring, then $Q(R)$ is Gaussian, since $Q(R)$ is a localization of $R$.

(e) If $R$ is a locally Prüfer ring, then $Q(R)$ is locally Prüfer.

To see this, we follow the proof from [10]: Let $Q$ be a prime ideal of $Q(R)$, and set $P=Q \cap R$. Since a locally Prüfer ring is Prüfer, we obtain that $Q(R)_{Q} \cong R_{P}$. Since $R$ is locally Prüfer, it follows that $Q(R)$ is locally Prüfer.

(f) If $R$ is a Prüfer ring, then $Q(R)$ is Prüfer, since a total ring of quotients has no proper regular ideals.

By imposing the desired Prüfer condition on the total ring of quotients, Bazzoni and Glaz [7] were able to specify when a Prüfer ring satisfies any of the other Prüfer conditions. Specifically, we have the following theorem.

Theorem 5.8 ([7]) Let $R$ be a Prüfer ring. Then:

(1) $R$ is a Gaussian ring if and only if $Q(R)$ is a Gaussian ring.

(2) $R$ is an arithmetical ring if and only if $Q(R)$ is an arithmetical ring.

(3) $\quad$ w. $\operatorname{dim} R \leq 1$ if and only if w. $\operatorname{dim} Q(R) \leq 1$.

(4) $R$ is a semihereditary ring if and only if $Q(R)$ is a semihereditary ring.

This theorem has turned out to be useful in certain investigations (see, for example, Sect. 7). Its proof is a corollary of the following theorem proved by Bazzoni and Glaz [7].

Theorem 5.9 ([7]) Let $R$ be a ring. Then:

(1) $R$ is an arithmetical ring if and only if $R$ is a Gaussian ring and $Q(R)$ is an arithmetical ring.

(2) $R$ is a Gaussian ring if and only if $R$ is a Prüfer ring and $Q(R)$ is a Gaussian ring.

Boynton [10] proves an analogous result for locally Prüfer rings.

Theorem 5.10 ([10]) The ring $R$ is locally Prüfer if and only if $R$ is Prüfer and $Q(R)$ is locally Prüfer.

In particular, we can add to Theorem 5.8: For the class of Prüfer rings, $R$ is a locally Prüfer ring if and only if $Q(R)$ is a locally Prüfer ring.

Recall that in Sect. 4 we numbered the six Prüfer conditions as follows:

(1) $R$ is a semihereditary ring.

(2) $\quad$ w. $\operatorname{dim} R \leq 1$.

(3) $R$ is an arithmetical ring.

(4) $R$ is a Gaussian ring.

(5) $R$ is a locally Prüfer ring.

(6) $R$ is a Prüfer ring.

As a corollary of Theorem 5.9 and Theorem 5.10 we can now fully describe the conditions on $Q(R)$ that allow for reversal of implications. Corollary 5.11 was proved in [7] for Prüfer conditions (1) through (4) and (6), and in [10] for Prüfer condition (5).

Corollary 5.11 ([7,10]) Let $R$ be a commutative ring.

(1) $R$ has Prüfer condition ( $n)$ if and only if $R$ has Prüfer condition $(n+1)$ and $Q(R)$ has Prüfer condition (n) for all $1 \leq n \leq 5$.

(2) If $Q(R)$ is von Neumann regular, then all the Prüfer conditions, (1) through (6), are equivalent.

To conclude this section, we pose an open question and three examples that highlight the difficulties.

Open Question 2 Let $R$ be a total ring of quotients. Under what conditions is $R$ a Gaussian ring? 
Example 5.12 A non-Gaussian total ring of quotients.

Let $k$ be a countable, algebraically closed field and let $I$ be an infinite set. Denote by $k^{I}$ the set of all maps from $I$ to $k$. In 1971, Quentel [48] constructed an algebra $R \subset k^{I \times \mathbb{N}^{\mathbb{N}}}$, where $\mathbb{N}$ denotes the natural numbers, with the following properties:

(1) $R$ is reduced.

(2) $R=Q(R)$

(3) $R$ is not von Neumann regular.

(4) $\operatorname{Min}(R)$ is compact.

One can show that $R$ is not Gaussian as follows. Assume that $R$ is Gaussian. Since a Gaussian reduced ring has weak global dimension at most one, this means that the ring must be PF. The PF condition coupled with compact minimal prime spectrum is equivalent to the PF condition coupled with a von Neumann regular total ring of quotients. It follows that $R$ is von Neumann regular, which contradicts the hypothesis.

Example $5.13 R$ is not Gaussian, but $Q(R)$ is Gaussian.

Let $R$ be a local Noetherian reduced ring which is not a domain. Such a ring is not Gaussian because if it were, it would have been a domain. Since $R$ is Noetherian, $\operatorname{Min}(R)$ is finite. $\operatorname{Set} \operatorname{Min}(R)=\left\{p_{1}, \ldots, p_{n}\right\}$ and thus $Q(R)=R_{p_{1}} \times \cdots \times R_{p_{n}}$, where each $R_{p_{i}}$ is a field. As $Q(R)$ is a finite direct product of fields, it is von Neumann regular, hence also Gaussian.

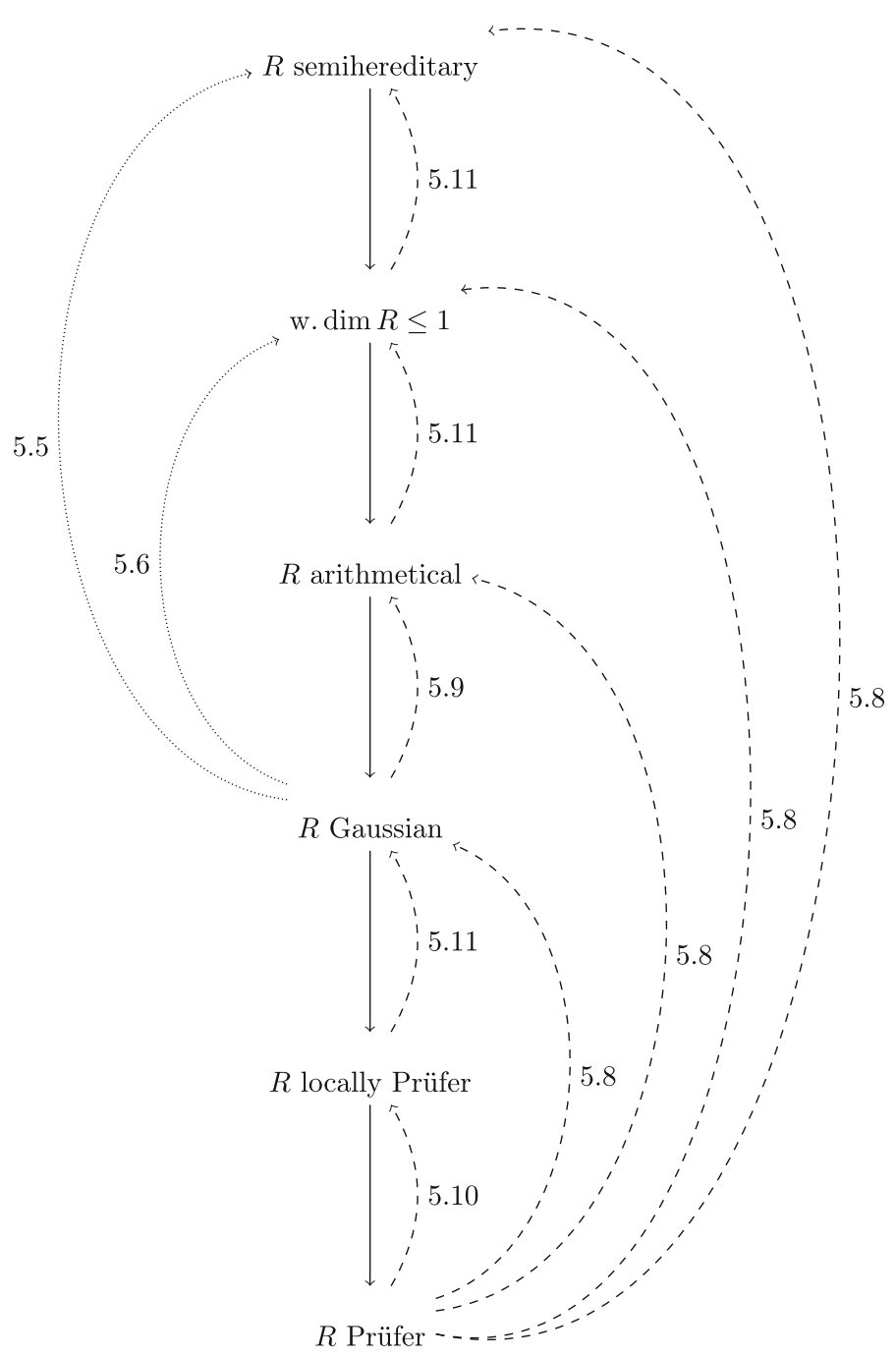


Example $5.14 R$ Gaussian and $Q(R)$ Gaussian, but $Q(R)$ is not von Neumann regular.

Any non-coherent ring $R$ with w. $\operatorname{dim} R=1$ has $Q(R)$ with w. $\operatorname{dim} Q(R) \leq 1$. Therefore $Q(R)$ is Gaussian, but if $Q(R)$ were von Neumann regular, then $R$ would be semihereditary and therefore coherent.

Using the results of this section, we can express the relations between the rings satisfying the various Prüfer conditions in the diagram on page 10. Here the dotted arrows represent implications from imposing zero divisor conditions while the dashed arrows are due to implications arising from conditions on the total ring of quotients.

\section{Homological Dimensions and Prüfer Conditions}

The connection between rings satisfying various Prüfer conditions and rings of w. $\operatorname{dim} R \leq 1$ raises some natural questions. For example, if $R$ is a Gaussian ring, what values can w. $\operatorname{dim} R$ take? The weak global dimension is not the only homological dimension that appears in investigations related to Prüfer conditions.

Definition 6.1 Denote by $\bmod R$ the set of all $R$-modules admitting a projective resolution consisting of finitely generated projective modules. The finitistic projective dimension of $R$, denoted fP. $\operatorname{dim} R$ is

$$
\text { fP. } \operatorname{dim} R=\sup \left\{\text { proj. } \operatorname{dim}_{R} M: M \in \bmod R \text { and proj. } \operatorname{dim}_{R} M<\infty\right\} .
$$

If $R$ is a coherent ring, then

$$
\text { fP. } \operatorname{dim} R=\sup \left\{\text { proj. } \operatorname{dim}_{R} M: M \text { is finitely presented and proj. } \operatorname{dim}_{R} M<\infty\right\} .
$$

The relation between the weak global dimension and the finitistic projective dimension of a ring is given by the following theorem, whose first part is immediate from the definition and whose second part was proved in [26].

Theorem 6.2 ([26]) Let $R$ be a commutative ring. Then:

(1) $\mathrm{fP} . \operatorname{dim} R \leq \mathrm{w} \cdot \operatorname{dim} R$.

(2) If $R$ is a local, coherent, regular ring, then $\mathrm{fP} . \operatorname{dim} R=\mathrm{w} \cdot \operatorname{dim} R$.

A ring $R$ is called regular if every finitely generated ideal of $R$ has finite projective dimension. Recall the classical definition of regularity for Noetherian rings: A local Noetherian ring $R$ is regular if its maximal ideal is generated by $n$ elements, where $n=\operatorname{Krull}$. $\operatorname{dim} R$. A general Noetherian ring $R$ is regular if $R$ is locally regular. It follows that for a Noetherian ring $R$ the two definitions of regularity coincide. Examples of non-Noetherian regular rings include all coherent rings of finite weak global dimension, but there are coherent rings of infinite weak global dimension, which are regular. For example, $R=k\left[x_{1}, x_{2}, \ldots\right]$, where $k$ is a field. More details about these types of rings may be found in [25].

The close relation between the finitistic projective dimension and the weak global dimension makes it natural to involve fP. $\operatorname{dim} R$ in any attempt to track the possible values of w. $\operatorname{dim} R$ for a $\operatorname{ring} R$ satisfying a Prüfer condition. Moreover, the finitistic projective dimension of certain rings satisfying a Prüfer condition seems to capture the "finite part" of its weak global dimension. That is, as we see in Theorems 6.3 and 6.5, the weak global dimension of coherent Gaussian rings is either equal to the finitistic projective dimension, and therefore less than or equal to 1 , or it is infinite.

\section{Prüfer conditions and the finitistic projective dimension}

Thus far, the only known results regarding the finitistic projective dimension of rings satisfying Prüfer conditions concern Gaussian rings. The following was proved by Glaz in [30].

Theorem 6.3 ([30]) If $R$ is a coherent Gaussian ring, then $\mathrm{fP} . \operatorname{dim} R \leq 1$. Moreover, if $R$ is a Gaussian local ring with maximal ideal $m$ consisting entirely of zero divisors, then $\mathrm{fP} . \operatorname{dim} R=0$.

In particular, coherence is not necessary for the last statement of Theorem 6.3. More recently, it has been shown by Bazzoni and Glaz [7] that coherence is unnecessary for the first implication of Theorem 6.3 as well.

Theorem 6.4 ([7]) If $R$ is a Gaussian ring, then $\mathrm{fP} . \operatorname{dim} R \leq 1$.

This leads to the following two questions. 
Open Question 3 If $R$ is a Prüfer ring, is $\mathrm{fP} \cdot \operatorname{dim} R \leq 1$ ?

As we have seen, the answer is affirmative for Gaussian rings. It is also true for Prüfer domains.

Open Question 4 If $R$ is a total ring of quotients, is $\mathrm{fP} . \operatorname{dim} R=0$ ?

Note that a total ring of quotients is always a Prüfer ring, so this question asks if for this particular kind of Prüfer ring, fP. $\operatorname{dim} R$ is not only at most 1, but actually equal to 0 . Theorem 6.3 shows that this is true for a local Gaussian total ring of quotients.

\section{Prüfer conditions and the weak global dimension}

Using the result on the finitistic projective dimension of Gaussian coherent rings, Glaz [30] determined the values of the weak global dimension of these rings.

Theorem 6.5 ([30]) Let $R$ be a coherent Gaussian ring. Then w. $\operatorname{dim} R=0,1$, or $\infty$. In particular, $R$ is either von Neumann regular, semihereditary, or of infinite weak global dimension.

Contrary to the finitistic projective dimension case, it has not been possible so far to completely remove the coherence restriction. Instead, Bazzoni and Glaz [7] succeeded in proving a similar result under a less restrictive Prüfer condition.

Theorem 6.6 ([7]) If $R$ is a coherent Prüfer ring, then w. $\operatorname{dim} R=0,1$, or $\infty$.

If the ring is in fact a total ring of quotients, this theorem can be refined.

Corollary 6.7 Let $R$ be a coherent total ring of quotients. Then $\mathrm{w}$. $\operatorname{dim} R=0$ or $\infty$. In particular, a coherent total ring of quotients is either von Neumann regular or has infinite weak global dimension.

This result can be proved as follows. Let $R=Q(R)$. By Theorem 6.6, w. $\operatorname{dim} R=0,1$ or $\infty$. If w. $\operatorname{dim} R \leq 1$ since $R$ is coherent, it is semihereditary, hence $Q(R)$ is von Neumann regular. This means that w. $\operatorname{dim} R=0$.

The following example shows that this result is no longer true if the coherence condition is removed.

Example 6.8 A non-coherent total ring of quotients $Q(R)$ with w. $\operatorname{dim} Q(R)=1$.

Consider a non-semihereditary $\operatorname{ring} R$ with w. $\operatorname{dim} R=1$. Then w. $\operatorname{dim} Q(R) \leq$ w. $\operatorname{dim} R \leq 1$, but if w. $\operatorname{dim} Q(R)=0$, then $Q(R)$ is von Neumann regular and therefore $R$ is semihereditary.

The following result by Bazzoni and Glaz [7] establishes the values of the weak global dimension of an arithmetical ring.

Theorem 6.9 ([7,46]) If $R$ is an arithmetical ring, then $\mathrm{w}$. $\operatorname{dim} R=0,1$, or $\infty$.

Osofsky [46] proved that a local, non-reduced ring with zero divisors, whose ideals are linearly ordered, has a module with infinite projective dimension. In [7], Bazzoni and Glaz proved the general case by a reduction to the case where they could use Osofsky's result.

Theorem 6.10 ([7]) If $R$ is a Gaussian ring admitting a maximal ideal $m$ such that the nilradical of $R_{m}$ is a nonzero nilpotent ideal, then $\mathrm{w} . \operatorname{dim} R=\infty$.

Some open questions concerning the possible values of the weak global dimensions of rings satisfying Prüfer conditions are the following.

Open Question 5 If $R$ is a Gaussian ring, is w. $\operatorname{dim} R=0,1$, or $\infty$ ?

We saw this to be the case for coherent Gaussian rings, arithmetical rings, and the strange Gaussian rings of the previous theorem.

Open Question 6 If $R$ is a total ring of quotients, is w. $\operatorname{dim} R=0,1$, or $\infty$ ?

This is true if $R$ is coherent, in which case w. $\operatorname{dim} R=0$ or $\infty$ and also holds for one example of a noncoherent ring (see Example 6.8). An answer to this question will yield a partial answer to the next question.

Open Question 7 What are the values of $\mathrm{w} . \operatorname{dim} R$ when $R$ is a Prüfer ring? 


\section{Prüfer Conditions in Pullback Rings}

The next step in the investigation of Prüfer conditions was to consider them in ring constructions. The first ring constructions to be considered were pullback rings, also called constructions of the type $D+M$. The classical $D+M$ construction is described in Gilmer's book [24] and according to Gilmer, goes back to Krull's work [37] in 1936. The evolution of these constructions went through three stages. We briefly describe these stages below:

\section{Krull's $D+M$ definition}

In [37] Krull defined $D+M$ rings as follows: Let $T=V$ be a valuation domain with maximal ideal $M$, along with a map $\phi: V \rightarrow K=V / M$. Let $D$ be a subring of $K$ and let $\phi^{-1}(D)=D_{1}$. Then $D_{1}$ has $M$ as a common ideal with $V$. Assume that $K \subset V$, so that $V=K+M$ and $D_{1}=D+M$.

This construction was investigated for various finiteness and homological properties by numerous researchers. We mention several articles related to the Prüfer conditions later in this section.

\section{Brewer and Rutter's $D+M$ definition}

In [11] Brewer and Rutter extended Krull's construction as follows: Let $T$ be a domain and let $K$ be a field which is a retract of $T$, that is, there exist maps $\phi: K \rightarrow T$ and $\psi: T \rightarrow K$ satisfying $\psi \circ \phi=1_{K}$. Then $T=K+M$, where $M$ is a maximal ideal of $T$. Let $D$ be a subring of $K$ and set $R=D+M$.

This general construction and the particular case where $T=K[x]=K+x K[x]$, with $K$ a field, $D$ a subring of $K$ such that $Q(D)=K$ and $R=D+x K[x]$, were investigated in [11]. Costa, Mott and Zafrullah [15] considered the case where $R=D+x D_{S}[x]$, where $D$ is a ring and $S$ is any multiplicatively closed subset of $D$. Before moving on to $D+M$ constructions that admit zero divisors, we reproduce the following result, which classifies when this $D+M$ construction is a Prüfer domain.

Theorem 7.1 ([11]) For the $D+M$ construction due to Brewer and Rutter, $R$ is a Prüfer domain if and only if $D$ and $T=K+M$ are Prïfer domains and $K=Q(D)$.

\section{Pullback rings}

In all of the above constructions, $T$ is a domain. Gabelli and Houston [21,22] considered Brewer and Rutter's $D+M$ constructions (with $T$ a domain), but viewed them as pullback rings. This alternative perspective allowed for the introduction of zero divisors.

The first authors to consider pullback rings with zero divisors were probably Greenberg and Vasconcelos (1974 [31], 1976 [32]). They aimed to classify rings of weak global dimension 2 and considered a very specialized conductor square

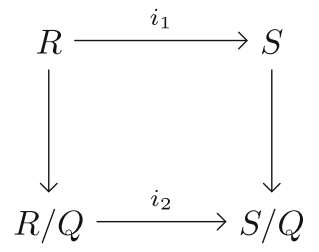

where $i_{1}$ is a flat epimorphism, $Q$ is a flat ideal of $R$, and $Q S=S$. Fontana (1980 [19]) and Cahen (1988 [13]) also considered a number of properties of pullback rings with zero divisors in a slightly less restrictive setting than the above conductor square. More recently, Prüfer conditions in pullback settings with zero divisors were considered by Bakkari and Mahdou (2009 [4]) as well as Boynton (2007 [8], 2008 [9], 2011 [10]).

The setting investigated by Bakkari and Mahdou [4] is as follows: $(T, M)$ is a local ring, not necessarily a domain, $T / M=K, T=K+M, K$ is field, $D$ is a subring of $K$ with $Q(D)=K$, and $R=D+M$. Using this construction, they obtained the following result.

Theorem 7.2 ([4]) Let $T, R, D$, and $M$ be as above. Then:

(1) $R$ is a semihereditary ring if and only if $T$ and $D$ are semihereditary rings.

(2) $\mathrm{w} \cdot \operatorname{dim} R \leq 1$ if and only if w. $\operatorname{dim} T \leq 1$ and $\mathrm{w} \cdot \operatorname{dim} D \leq 1$.

(3) $R$ is an arithmetical ring if and only if $T$ and $D$ are arithmetical rings.

(4) $R$ is a Gaussian ring if and only if $T$ and $D$ are Gaussian rings.

(5) $R$ is a Prüfer ring if and only if $T$ and $D$ are Prüfer rings. 
As a consequence of this theorem, they found a host of examples of rings satisfying some Prüfer conditions but not others.

In his work, Boynton [8-10] considered a more general pullback setting called a conductor square. Specifically, $R$ is a subring of a ring $T$, and assume that they have a common non-zero conductor ideal. Let $C$ be the largest such ideal and set $A=R / C$ and $B=T / C$. The following "conductor square" commutes with the usual maps ( $b$ and $\eta_{1}$ are projection maps into quotient rings and $i_{1}$ is an inclusion map):

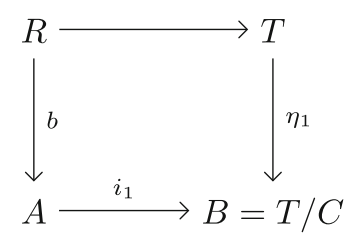

Note that $C=\operatorname{ker} \eta_{1}$. If $C$ is a regular ideal then this conductor square is called a regular conductor square. In this setting, Boynton $[9,10]$ determined conditions for $R$ to satisfy all six Prüfer conditions.

Theorem 7.3 ([9,10]) Consider the regular conductor square setting above. Then:

(1) $R$ is a semihereditary ring if and only if $T$ is a semihereditary ring, $A_{p}$ is a Prüfer ring, and $B_{p}$ is an overring of $A_{p}$ for each prime (maximal) ideal $p$ of $R$.

(2) $R$ satisfies w. $\operatorname{dim} R \leq 1$ if and only if $T$ satisfies $\mathrm{w} . \operatorname{dim} T \leq 1, A_{p}$ is a Prüfer ring, and $B_{p}$ is an overring of $A_{p}$ for each prime (maximal) ideal $p$ of $R$.

(3) $R$ is an arithmetical ring if and only if $T$ is an arithmetical ring, $A_{p}$ is a Prüfer ring, and $B_{p}$ is an overring of $A_{p}$ for each prime (maximal) ideal $p$ of $R$.

(4) $R$ is a Gaussian ring if and only if $T$ is a Gaussian ring, $A_{p}$ is a Prüfer ring, and $B_{p}$ is an overring of $A_{p}$ for each prime (maximal) ideal $p$ of $R$.

(5) $R$ is locally Prüfer if and only if $T_{p}$ is a Prüfer ring for every prime ideal $p$ of $R, A$ is locally Prüfer, and $B_{p}$ is an overring of $A_{p}$ for every prime ideal $p$ of $R$.

(6) If $R$ is a Prüfer ring, then $A$ and $T$ are Prüfer rings and $B_{p}$ is an overring of $A_{p}$ for each prime ideal $p$ of $R$. Conversely, for every prime ideal $p$ of $R$, if $A$ is locally Prüfer, $T_{p}$ is a Prüfer ring, and $B_{p}$ is an overring of $A_{p}$, then $R$ is a Prüfer ring.

As far as Prüfer conditions go, we seem to have a good answer in a general pullback setting, although it would be nice to explore the situation when the conductor ideal is not necessarily regular. Nevertheless, even in the regular conductor square setting, some open questions arise. We have conditions for $R$ to have w. $\operatorname{dim} R \leq 1$, but we do not have conditions linking w. $\operatorname{dim} R$ with w. $\operatorname{dim} T$, w. $\operatorname{dim} A$, or w. $\operatorname{dim} B$ even in the most classical domain $D+M$ construction. The papers considering homological dimensions of pullback rings, [17] and [31], investigate only gl. $\operatorname{dim} R$ in domains, and do this in two specific $D+M$ settings.

We remark that another property that was only partially explored in $D+M$ constructions is coherence. Partial answers regarding when a pullback ring is coherent were provided only for special cases of domains (see $[11,18,21])$.

Open Question 8 Given any of the pullback settings above, is it possible to find formulas and conditions that yield a connection between $\mathrm{w} . \operatorname{dim} R$ and the weak global dimension of the other rings involved? Also, when is such a pullback ring coherent?

\section{Prüfer Conditions in Localizations of Polynomial Rings}

A different kind of ring construction which came to prominence in Quillen's solution to Serre's conjecture [49] and its "companion" construction are two localizations of a polynomial ring in one variable.

Definition 8.1 Let $R$ be a ring and set $U=\{f \in R[x]: f$ is monic $\}$ and $V=\{f \in R[x]: c(f)=R\}=$ $R[x]-\cup_{m \in \operatorname{Max} R} m R[x]$. Then $U$ and $V$ are multiplicatively closed subsets of $R[x]$. Let $R\langle x\rangle=R[x]_{U}$ and $R(x)=R[x]_{V}$.

We note that $R[x] \subset R\langle x\rangle \subset R(x)$, and $R(x)$ is a localization of $R\langle x\rangle$. In addition, both $R(x)$ and $R\langle x\rangle$ are faithfully flat over $R$. These localizations of $R[x]$ were thoroughly investigated by a number of authors 
(see [1] and [25] for a survey of results concerning these rings). We mention below those results that shed some light on the ascent and descent of the six Prüfer conditions between $R$ and $R(x)$ or $R\langle x\rangle$.

The result below was proved by Glaz [25] for $R(x)$ and by Le Riche [38] for $R\langle x\rangle$.

Theorem 8.2 ([25,38]) Let $R$ be a commutative ring. Then:

(1) $R(x)$ is semihereditary if and only if $R$ is semihereditary.

(2) $R\langle x\rangle$ is semihereditary if and only if $R$ is semihereditary and has Krull dimension at most one.

In 1989, Glaz [25] established the connection between w. $\operatorname{dim} R(x)$ (respectively, w. $\operatorname{dim} R\langle x\rangle$ ) and w. $\operatorname{dim} R$, when $R$ is a stably coherent ring of finite weak global dimension. But in general, the connection between the weak global dimensions is not known. In the context of Prüfer conditions this leads to another open question.

Open Question 9 Under what conditions on $R$ and $\mathrm{w} . \operatorname{dim} R$ will w. $\operatorname{dim} R(x)($ respectively, w. $\operatorname{dim} R\langle x\rangle)$ be at most one?

The arithmetical case was resolved by Anderson D.D., Anderson D.F., and Markanda in [1].

Theorem 8.3 ([1]) Let $R$ be a commutative ring. Then:

(1) $R(x)$ is arithmetical if and only if $R$ is arithmetical. Moreover, in this case, $R(x)$ is Bezout, i.e. every finitely generated ideal of $R$ is principal.

(2) $R\langle x\rangle$ is arithmetical if and only if $R$ is arithmetical with Krull dimension at most one and if $p \subsetneq q$ are prime ideals of $R$, then $R_{p}$ is a field.

Given the criteria for $R(x)$ and $R\langle x\rangle$ to be semihereditary or arithmetical, it is natural to ask when these rings are Gaussian.

Open Question 10 Under what conditions on $R$ will $R(x)$ (respectively, $R\langle x\rangle$ ) be Gaussian?

In order to characterize $R(x)$ and $R\langle x\rangle$ as Prüfer rings, Anderson D.D., Anderson D.F., and Markanda [1] considered another Prüfer condition.

Definition 8.4 A ring $R$ is strongly Prüfer if every finitely generated ideal $I$ of $R$ with $(0: I)=0$ is locally principal.

By Theorem 2.3, a Gaussian ring satisfies this condition, so we have the following containments:

$$
\text { Gaussian rings } \subset \text { Strongly Prüfer rings } \subset \text { Prüfer rings. }
$$

It is still unclear where the strongly Prüfer condition falls relative to the locally Prüfer condition. Huckaba's book [35] provides several examples of Prüfer rings that are not strongly Prüfer (one is a total ring of quotients). Lucas [41] clarified the relation between the strongly Prüfer condition of a ring $R$ and the strongly Prüfer condition of it total ring of quotients.

Theorem 8.5 ([41]) Let $R$ be a commutative ring. Then $R$ is a strongly Prüfer ring if and only if $R$ is a Prüfer ring and $Q(R)$ is a strongly Prüfer ring.

In particular this theorem adds to Theorem 5.8: For the class of Prüfer rings, $R$ is strongly Prüfer if and only if $Q(R)$ is strongly Prüfer. The similarity of behavior of the strongly Prüfer condition relative to its total ring of quotients to the other six Prüfer conditions described in this article raises an interesting question regarding pullback situations. To summarize:

Open Question 11 How does the pullback situation behave for the strongly Prüfer condition? Also, how do the locally Prüfer condition and the strongly Prüfer condition relate to each other?

Using the notion of strongly Prüfer rings, Anderson D.D., Anderson D.F., and Markanda [1] determined when $R(x)$ and $R\langle x\rangle$ are Prüfer rings.

Theorem 8.6 ([1]) Let $R$ be a commutative ring. Then:

(1) $R(x)$ is a Prüfer ring if and only if $R$ is a strongly Prüfer ring.

(2) $R\langle x\rangle$ is a Prüfer ring if and only if $R$ is a strongly Prüfer ring, the Krull dimension of $R$ is at most 1, and if $p \subsetneq q$ are prime ideals of $R$, then $R_{p}$ is a field. 
We note that both $R(x)$ and $R\langle x\rangle$ satisfy the following property: If $I$ is a finitely generated ideal which contains only zero divisors, then $I$ has a non-zero annihilator (this property is called "Property A" in [35]). It follows immediately from Theorem 3.9 that a ring satisfying this property is Prüfer if and only if it is strongly Prüfer. Therefore, both $R(x)$ and $R\langle x\rangle$ are Prüfer rings if and only if they are strongly Prüfer rings. To complete the picture for the Prüfer conditions of $R(x)$ and $R\langle x\rangle$, the locally Prüfer condition must also be investigated.

\section{Open Question 12 Under what conditions on $R$ will $R(x)$ (respectively, $R\langle x\rangle$ ) be locally Prüfer?}

Acknowledgments The authors thank both referees for numerous useful comments that improved the content and the presentation of this article. The first author gratefully acknowledges the gracious hospitality of the algebra group at the Università di Roma Tre during her three month sabbatical visit in 2010. A significant portion of this article was prepared during that period.

\section{References}

1. Anderson, D.D.; Anderson, D.F.; Markanda R.: The rings $R(X)$ and $R\langle X\rangle$. J. Algebra 95, 96-115 (1985)

2. Anderson, D.D.; Kang, B.G.: Content formulas for polynomials and power series and complete integral closure. J. Algebra 181, 82-94 (1996)

3. Arnold, J.; Gilmer R.: On the contents of polynomials. Proc. Am. Math. Soc. 24, 556-562 (1970)

4. Bakkari, C.; Mahdou, N.: Prüfer-like conditions in pullbacks. In: Commutative Algebra and Applications, pp. 41-47. Walter de Gruyter, Berlin (2009)

5. Bakkari, C.; Kabbaj, S.; Mahdou, N.: Trivial extensions defined by Prüfer conditions. J. Pure Appl. Algebra 214, 53-60 (2010)

6. Bazzoni, S.; Glaz, S.: Prüfer rings. In: Multiplicative Ideal Theory in Commutative Algebra, pp. 55-72. Springer, Berlin (2006)

7. Bazzoni, S.; Glaz, S.: Gaussian properties of total rings of quotients. J. Algebra 310, 180-193 (2007)

8. Boynton, J.: Pullbacks of arithmetical rings. Comm. Algebra 35, 2671-2684 (2007)

9. Boynton, J.: Pullbacks of Prüfer rings. J. Algebra 320, 2559-2566 (2008)

10. Boynton, J.: Local Prüfer properties of the total quotient ring. (to appear)

11. Brewer, J.; Rutter, E.: $D+M$ constructions with general overrings. Mich. Math. J. 23, 33-42 (1976)

12. Butts, H.S.; Smith, W.: Prüfer rings. Math. Z. 95, 196-211 (1967)

13. Cahen, P.-J.: Couples d'anneaux partageant un ideal. Arch. Math. (Basel) 51, 505-514 (1988)

14. Cartan, H.; Eilenberg, S.: Homological algebra. Princeton University Press, Princeton (1956)

15. Costa, D.; Mott, J.; Zafrullah, M.: The construction $D+X D_{s}[X]$. J. Algebra 53, 423-439 (1978)

16. Costa, D.; Mott, J.; Zafrullah, M.: Overrings and dimensions of general $D+M$ constructions. J. Nat. Sci. Math. 26, 7-13 (1986)

17. Dobbs, D.: On the global dimension of D M. Can. Math. Bull. 18, 657-660 (1975)

18. Dobbs, D.; Papick, I.: When is $D+M$ coherent? Proc. Am. Math. Soc. 56, 51-54 (1976)

19. Fontana, M.: Carrés cartésiens, anneaux divisés et anneaux localement divisés. Prépublication de l'Univ. de Paris-Nord 21 (1980)

20. Fuchs, L.: Über die Ideale arithmetischer Ringe. Math. Helv. 23, 334-341 (1949)

21. Gabelli, S.; Houston, E.: Coherent-like conditions in pullbacks. Mich. Math. J. 44, 99-123 (1997)

22. Gabelli, S.; Houston, E.: Ideal theory in pullbacks. In: Non-Noetherian Commutative Ring Theory, pp. 199-227. Math. Appl., vol. 520. Kluwer, Dordrecht (2000)

23. Gilmer, R.: Some applications of the Hilfssatz von Dedekind-Mertens. Math. Scand. 20, 240-244 (1967)

24. Gilmer, R.: Multiplicative ideal theory. Queen's papers on Pure Appl. Math., vol. 12 (1968)

25. Glaz, S.: Commutative coherent rings. Lecture Notes in Mathematics, vol. 1371. Springer, Berlin (1989)

26. Glaz, S.: On the coherence and weak dimension of the rings $R\langle x\rangle$ and $R(x)$. Proc. Am. Math. Soc. 106, $579-587$ (1989)

27. Glaz, S.; Vasconcelos, W.V.: Gaussian polynomials. In: Marcel Dekker Lecture Notes 186, 325-337 (1997)

28. Glaz, S.; Vasconcelos, W.V.: The content of Gaussian polynomials. J. Algebra 202, 1-9 (1998)

29. Glaz, S.: Prüfer conditions in rings with zero-divisors. In: Lecture Notes in Pure Appl. Math., vol. 241, 272-281. CRC Press (2005)

30. Glaz, S.: The weak dimension of Gaussian rings. Proc. Am. Math. Soc. 133, 2507-2513 (2005)

31. Greenberg, B.: Global dimension of cartesian squares. J. Algebra 32, 31-43 (1974)

32. Greenberg, B.; Vasconcelos, W.V.: Coherence of polynomial rings. Proc. Am. Math. Soc. 54, 59-64 (1976)

33. Griffin, M.: Prüfer rings with zero-divisors. J. Reine Angew Math. 239/240, 55-67 (1970)

34. Heinzer, W.; Huneke, C.: Gaussian polynomials and content ideals. Proc. Am. Math. Soc. 125, 739-745 (1997)

35. Huckaba, J.: Commutative rings with zero divisors. Pure and Applied Mathematics, vol. 117. Marcel Dekker, New York (1988)

36. Jensen, C.: Arithmetical rings. Acta Math. Hungr. 17, 115-123 (1966)

37. Krull, W.: Beiträge zur arithmetik kommutativer Integritätsbereiche. Math. Z. 41, 545-577 (1936)

38. Le Riche, L.: The ring $R\langle X\rangle$. J. Algebra 67, 327-341 (1980)

39. Loper, K.A.; Roitman, M.: The content of a Gaussian polynomial is invertible. Proc. Am. Math. Soc. 133, 1267-1271 (2005)

40. Lucas, T.: Some results of Prüfer rings. Pac. J. Math. 124, 333-343 (1986)

41. Lucas, T.: Strong Prüfer rings and the ring of finite fractions. J. Pure Appl. Algebra 84, 59-71 (1993)

42. Lucas, T.: Gaussian polynomials and invertibility. Proc. Am. Math. Soc. 133, 1881-1886 (2005)

43. Lucas, T.: The Gaussian property for rings and polynomials. Houston J. Math. 34, 1-18 (2008) 
44. Marot, J.: Sur le anneaux universellement Japonais. Ph.D. Thesis, Universite de Paris-Sud (1977)

45. Matlis, E.: The minimal prime spectrum of a reduced ring. Ill. J. Math. 27, 353-391 (1983)

46. Osofsky, B.: Global dimension of commutative rings with linearly ordered ideals. J. Lond. Math. Soc. 44, 183-185 (1969)

47. Prüfer, H.: Untersuchungen über Teilbarkeitseigenshaften in Körpern. J. Reine Angew. Math. 168, 1-36 (1932)

48. Quentel, Y.: Sur la compacité du spectre minimal d'un anneau. Bull. Soc. Math. France 99, 265-272 (1971)

49. Quillen, D.: Projective modules over polynomial rings. Invent. Math. 36, 167-171 (1976)

50. Rotman, J.: An introduction to homological algebra. Pure and Applied Mathematics, vol. 25. Academic Press, New York (1979)

51. Tsang, H.: Gauss's lemma. Ph.D. Thesis, University of Chicago (1965) 